\title{
Identification of in Vitro Resistance of Pigeon Peas [Cajanus Cajan (L.) Millsp.] to Phytophthora Stem Canker. ${ }^{1}$
}

\author{
R. Rodríguez and P. L. Meléndez $z^{2}$
}

\begin{abstract}
A total of 617 accessions and 40 pigeon pea lines were screened in vitro for their resistance to Phytophthora parasitica. Several entries were found resistant to the pathogen in stem-piece inoculations. Even after injury accessions 394-014, 396-383, 396-356, 396-731, 396-295 and lines 8 AB-2 and $731 \mathrm{BD}$ did not show lesions from $P$. parasitica even when injured.
\end{abstract}

\section{INTRODUCTION}

Pigeon pea stem canker caused by $P$. parasitica was first reported in Puerto Rico by Kaiser and Meléndez in 1978 (1). The disease is characterized by sunken dark brown to black lesions which may be present on the main stem as well as on the branches of infected plants. Losses result from poor stands because affected stems are girdled or become debilitated and break at canker sites or wilt above infection sites.

Pat et al. (3) and Williams et al. $(5,6)$ have reported a similar disease which is serious in Indian pigeon pea growing areas. In the northwestern region of Puerto Rico a greater disease incidence occurs in August, thus attacking the plants almost in the middle of their growing cycle (2).

One effective method to control Phytophthora stem canker will be the use of resistant varieties. To meet with this objective, local advanced lines, introductions and accessions from the germplasm collection were evaluated for their resistance to Phytophthora stem canker.

\section{MATERIALS AND METHODS}

The 617 accessions and 40 advanced lines were screened as follows: six $10-\mathrm{cm}$ stem pieces were collected from the main axis of 4 -month old plants grown in the greenhouse. Half of these were wounded artificially; the other half were left undisturbed. All stem-pieces were washed with $10 \%$ Lysol and the detergent Alconox. After washing in tap water, they were immersed for $5 \mathrm{~min}$ in each of the following solutions, $20 \%$ Clorox, $70 \%$ ethyl alcohol and finally rinsed in sterile distilled water. The stempieces were placed in petri dishes $(150 \times 20 \mathrm{~mm})$ containing sterile moist filter paper and exposed to PDA discs $(6 \mathrm{~mm})$ with hyphal tips obtained from a 15-day-old culture of $P$. parasitica isolated from cankered pigeon

\footnotetext{
${ }^{1}$ Submitted to Editorial Board December 20, 1983.

${ }^{2}$ Research Assistant and Phytopathologist, Crop Protection Department, College of Agricultural Sciences. University of Puerto Rico, Mayagüez, P.R.
} 

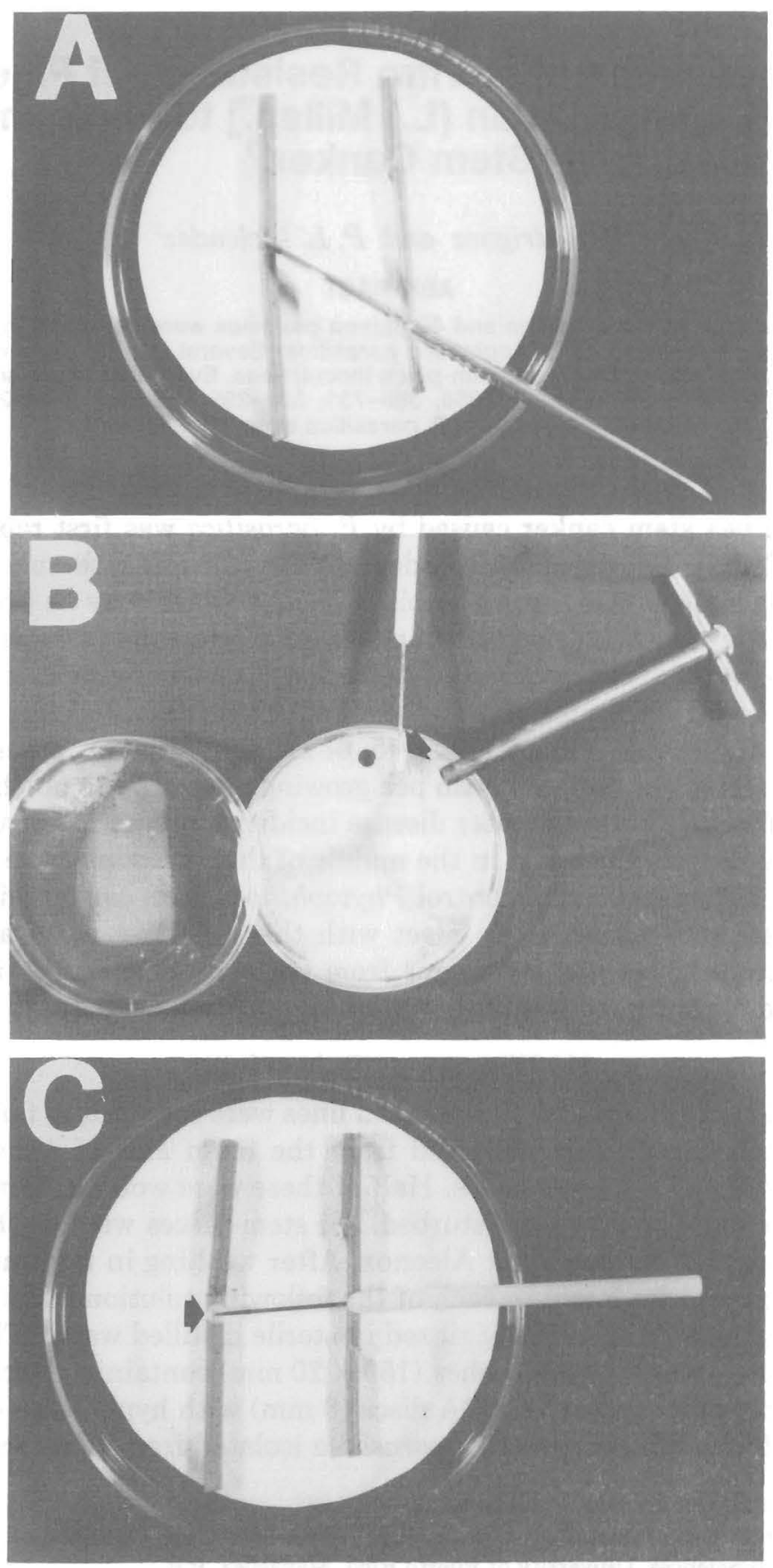

Fig. 1.-Inoculation steps for stem-piece technique. A. Artificial wounds. B. PDA discs with hyphal tips. C. Inoculation of the stem-pieces with fungus hyphal tips in direct contact with epidermal tissues. 
pea in the field (fig. 1.). Checks were similarly treated and exposed to PDA-discs free of the fungus. To test pathogen virulence, we included in each assay an additional check consisting of stem pieces of susceptible $\mathrm{cv}$. Kaki. After 4 days of incubation at $28^{\circ} \mathrm{C}$, the inoculated stem-pieces were examined on the basis of appearance of symptoms, and the extent of fungal invasion was measured and recorded.

\section{RESULTS AND DISCUSSION}

Pigeon pea accessions 394-014, 396-295, 396-356, 396-383 and 396731 did not show evidence of invasion by $P$. parasitica even when they were wounded. One hundred sixty-four accessions showed no external disease symptoms on unwounded inoculated stems; however, a range of lesion sizes was found with wound inoculations. Less than $20 \%$ of invasion was observed in 70 accessions and less than $40 \%$ was found in 41 others. Stem-pieces of 48 accessions showed invasion in more than $40 \%$ of their exposed surfaces (table 1). Two of the $P$. parasitica resistant accessions, 394-002 and 394-005, have also been found resistant to foliar diseases in Puerto Rico (4).

Several of the accessions evaluated reacted differently from the accessions mentioned above (table 2). Some of these lines showed symptoms when unwounded, but invasion was somewhat arrested compared with that showed on susceptible cv. Kaki. Conversely, accessions 394-279, 394-409, 394-675 394-712, 394-724, 394-768, 394-769 and 394-777 succumbed entirely to $P$. parasitica attack, showing cankers all over their exposed surfaces even when unwounded.

Tables 3 and 4 show the results on the evaluation of advanced lines and introductions. Line 8 AB-2 and 731BD did not show Phytophthora stem lesions even when wound inoculated. Twenty-five lines were not invaded by the pathogen when wounded, but they showed various extensions of lesions with wound inoculations. Lines 1 and 7221 particularly showed outstanding resistance to the pathogen even when wounded (table 3), whereas 7018 and 7172 were found susceptible to the disease, showing symptoms in more than 50\% of their stem-pieces (table 4). Line 64-16A, which possesses a certain degree of resistance to $P$. parasitica, has also been resistant to foliar diseases (4). Some advanced lines and introductions showed good levels of resistance to $P$. parasitica judging by the degree of infection under both wounded and unwounded conditions. Symptoms on unwounded and wounded stem-pieces from lines Kaki $\times$ GI 27-4A, 2624, 28, 6520, 6997 and 7181 covered less than $10 \%$ and $20 \%$ of their surfaces, respectively.

This screening has identified sources of resistance to P. parasitica, which can be used to improve commercial cultivars. Accessions and 
TABLE 1.-Percentage of surface cankered on wounded stem pieces from pigeon pea accessions after in vitro exposure to Phytophthora parasitica ${ }^{1}$

\begin{tabular}{|c|c|c|c|c|c|c|}
\hline \multicolumn{7}{|c|}{ Accession number ${ }^{2}$} \\
\hline $0 \%$ & $1-10 \%$ & $11-20 \%$ & $21-30 \%$ & $31-40 \%$ & $41-50 \%$ & $51-100 \%$ \\
\hline $394-014$ & 394-002 & 394-028 & 394-003 & 394-001 & 394-013 & 394-008 \\
\hline $396-295$ & 394-005 & 394-031 & 394-007 & 394-021 & $394-128$ & 394-039 \\
\hline $396-356$ & 394-012 & $394-065$ & 394-059 & $394-033$ & $394-170$ & 394-089 \\
\hline $396-383$ & $394-056$ & 394-069 & $394-206$ & $394-121$ & $394-280$ & $394-113$ \\
\hline $396-731$ & $394-152$ & $394-086$ & $394-220$ & $394-138$ & 394-306 & $394-123$ \\
\hline & 394-198 & 394-096 & $394-223$ & $394-174$ & $394-498$ & $394-237$ \\
\hline & $394-208$ & $394-103$ & $394-253$ & $394-260$ & $394-660$ & $394-275$ \\
\hline & $394-216$ & $394-114$ & $394-277$ & $394-273$ & $394-782$ & 394-706 \\
\hline & $394-255$ & $394-125$ & $394-294$ & $394-342$ & $394-783$ & $394-738$ \\
\hline & $394-274$ & $394-136$ & $394-301$ & $394-764$ & $394-784$ & $394-785$ \\
\hline & $394-313$ & $394-156$ & $394-302$ & $394-778$ & $394-788$ & $394-786$ \\
\hline & $394-314$ & $394-163$ & $394-401$ & $394-855$ & $396-023$ & $394-808$ \\
\hline & $396-240$ & $394-177$ & $394-490$ & $394-877$ & $396-046$ & $394-856$ \\
\hline & $396-313$ & $394-181$ & $394-656$ & $394-878$ & $396-159$ & $394-857$ \\
\hline & $396-745$ & $394-183$ & $394-725$ & $394-907$ & $396-180$ & $394-881$ \\
\hline & $396-784$ & $394-186$ & $394-766$ & 396-139 & $396-278$ & $396-015$ \\
\hline & $396-851$ & $394-194$ & $394-826$ & $396-282$ & $396-287$ & 396-042 \\
\hline & $396-939$ & 394-195 & $394-937$ & $396-863$ & $396-338$ & $396-128$ \\
\hline & & $394-202$ & $396-013$ & $396-982$ & $396-565$ & $396-203$ \\
\hline & & $394-204$ & $396-036$ & & $396-945$ & $396-318$ \\
\hline & & $394-205$ & $396-832$ & & $396-997$ & $396-840$ \\
\hline & & $394-212$ & $396-835$ & & & $397-158$ \\
\hline & & $394-215$ & & & & $397-273$ \\
\hline & & $394-219$ & & & & $397-341$ \\
\hline & & $394-225$ & & & & $397-534$ \\
\hline & & $394-226$ & & & & $397-985$ \\
\hline & & $394-256$ & & & & $397-998$ \\
\hline & & $394-258$ & & & & \\
\hline & & $394-262$ & & & & \\
\hline & & $394-272$ & & & & \\
\hline & & $394-276$ & & & & \\
\hline & & $394-282$ & & & & \\
\hline & & $394-286$ & & & & \\
\hline & & $394-290$ & & & & \\
\hline & & $394-315$ & & & & \\
\hline & & $394-321$ & & & & \\
\hline & & $394-353$ & & & & \\
\hline & & $394-494$ & & & & \\
\hline & & $394-528$ & & & & \\
\hline & & 396-005 & & & & \\
\hline & & $396-026$ & & & & \\
\hline & & $396-073$ & & & & \\
\hline & & $396-082$ & & & & \\
\hline & & $396-100$ & & & & \\
\hline & & $396-156$ & & & & \\
\hline & & $396-193$ & & & & \\
\hline & & $396-211$ & & & & \\
\hline & & $396-303$ & & & & \\
\hline & & $396-341$ & & & & \\
\hline & & $396-635$ & & & & \\
\hline & & $396-699$ & & & & \\
\hline & & $396-946$ & & & & \\
\hline
\end{tabular}

Inded cuttings frc 
TABLE 2.- Selection of pigeon pea accessions based on percentage of surface cankered by Phytophthora parasitica

\begin{tabular}{lllll}
\hline \multicolumn{5}{c}{ Accession number } \\
\hline $\begin{array}{c}\text { Less than 10\% when unwounded } \\
\text { and less than 20\% when } \\
\text { wounded }\end{array}$ & \multicolumn{3}{c}{ More than $50 \%$ of invasion under both condi- } \\
tions \\
\hline $394-094$
\end{tabular}

${ }^{1}$ USDA-PIN. Accessions received through S-9 Regional Project.

${ }^{2}$ Almost $100 \%$ of invasion in both conditions.

TABLE 3.-Percentage of surface cankered on wounded stem pieces from pigeon pea advanced lines and introductions after in vitro exposure to P. parasitica ${ }^{1}$

\begin{tabular}{|c|c|c|c|c|c|}
\hline \multicolumn{6}{|c|}{ Line } \\
\hline $0 \%$ & $1-10 \%$ & $11-20 \%$ & $21-30 \%$ & $31-40 \%$ & $41-50 \%$ \\
\hline $\begin{array}{l}8 \mathrm{AB}-2 \\
73-1 \mathrm{BD}\end{array}$ & $\begin{array}{l}1 \\
7221\end{array}$ & $\begin{array}{l}64-16 \text { A } \\
69-68 \\
1641 \\
3 D-8102\end{array}$ & $\begin{array}{l}\text { Am } \times \text { Kaki } \\
69-58 \\
\text { Cv. 2-B-Bushy } \\
64-21 \text { B-2 } \\
\text { Pusa Ageti } \\
\text { 3D-8103 } \\
\text { OP61 } \\
\text { UPA } 120\end{array}$ & $\begin{array}{l}\text { 16 AIrr } \\
142 \mathrm{~A} \\
4779 \\
\text { OP62 } \\
\text { T21 } \\
\text { B51 } \\
\text { 3D-8125 }\end{array}$ & $\begin{array}{l}69-52 \\
\text { Pant-A-2 }\end{array}$ \\
\hline
\end{tabular}

${ }^{1}$ Unwounded stem pieces from each line were not invaded by the pathogen.

advanced lines which have shown no $P$. parasitica lesions, even when wound inoculated are particularly promising. However, lines which resisted invasion when unwounded may not be as valuable for increasing disease resistance because in the field, crops are subjected to a wide 
number of factors which might affect the integrity of epidermal stem tissues. Consequently, lines and accessions with certain levels of resistance when injured should be considered in breeding for resistance to Phytophthora stem canker disease, particularly if they also have superior agronomic traits. Accessions and advanced lines have been identified with combined resistance to Phytophthora stem canker, rust and foliar spots in Puerto Rico. These lines can be important sources of multiple disease resistance.

The stem-piece technique is a useful tool in screening for resistance. It provides conditions for rapid lesion development by permitting early detection of disease differences among pigeon pea lines. Heterogenicity of test materials complicates their classification. Thus, accessions selected under these conditions should be further evaluated to confirm their disease reactions under field conditions.

TABLE 4.-Distribution of advanced lines and introductions based on percentage of surface cankered by P. parasitica on wounded and unwounded stem pieces

\begin{tabular}{lcc}
\hline $\begin{array}{c}\text { Less than } 10 \% \text { when unwounded } \\
\text { and less than } 50 \% \text { when } \\
\text { wounded }\end{array}$ & $\begin{array}{c}\text { Less than } 20 \% \text { when unwounded } \\
\text { and less than } 50 \% \text { when } \\
\text { wounded }\end{array}$ & $\begin{array}{c}\text { More than } 50 \% \text { of invasion } \\
\text { under both conditions }\end{array}$ \\
7 & 7119 & 7018 \\
Kaki $\times$ GI27-4A & 7135 & 7172 \\
12 & 7229 & \\
2624 & 7035 & \\
28 & & \\
6520 & & \\
6997 & & \\
7181 & & \\
\hline
\end{tabular}

\section{RESUMEN}

So evaluó un total de 617 líneas de la colección de germoplasma y 40 lineas introducidas y desarrolladas por el programa de fitomejoramiento de gandul para resistencia al cancro causado por Phytophthora. La evaluación de llevó a cabo in vitro utilizando el método de pedazos de tallos. Algunos de los materiales evaluados mostraron poseer resistencia al patógeno. Las líneas 394-014, 396-383, 396-356, 396-731, 396-295, $8 \mathrm{AB}-2$ y $731 \mathrm{BD}$ no mostraron síntomas de la enfermedad aun en presencia de heridas. Diferentes grados de resistencia fueron detectados entre la población de plantas examinadas.

\section{LITERATURE CITED}

1. Kaiser, W. J. and Meléndez P. L., 1978. A Phytophthora stem canker disease of pigeon pea in Puerto Rico, Plant Dis. Rep. 62: 240-42. 
2. Lebrón-López, Ligia E., 1981. Epidemiologia y control del cancro del tallo del gandul (Cajanus cajan (L.) Millsp.) causado por Phytophthora parasitica Dast.; M.S. Thesis, College of Agricultural Sciences, University of Puerto Rico, Mayagüez, P.R.

3. Pal, M., J. S. Grewal and Sarbhoy A. K., 1970. A new stem rot of arhar caused by Phytophthora, Indian Phytopathology 23: 583-87.

4. Rodriguez, Rocio and Meléndez P. L., 1984. Field Screening pigeon pea (Cajanus cajan) for resistance to foliar diseases in Puerto Rico, J. Agric. Univ. P. R. 68 (3); 275-80.

5. Williams, F. J., Amin K. S., and Baldev B., 1975. Phytophthora stem blight of Cajanus cajan, Phytopathology 65: 1029-030.

6. — Grewal J. S., and Amin K. S., 1968. Serious and new disease of pulse crops in India 1966, Plant Dis. Rep. 52: 300-04. 